\title{
Efeito Transiente de Exercícios de Flexibilidade na Articulação do Quadril Sobre a Marcha de Idosas
}

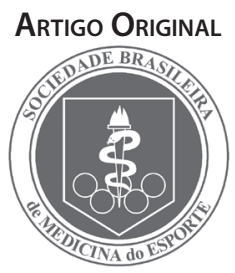

Transient Effect of Flexibility Exercises in the Hip Joint on the Gait of Older Women

\author{
Fabiano Cristopoliski ${ }^{1}$ \\ Thiago Augusto Sarraf ${ }^{1}$ \\ Valério Henrinque Dezan ${ }^{1}$ \\ Cléver Luiz Gregolin Provensi ${ }^{1}$ \\ André Luiz Félix Rodacki ${ }^{1}$ \\ 1. Departamento de Educação \\ Física, Universidade Federal do \\ Paraná, Curitiba, PR.
}

\section{Endereço para correspondência:} Fabiano Cristopoliski

Av. Vereador Toaldo Túlio, 3465, São Braz, Curitiba, PR

CEP 82300-000

Email: fabianoufpr@yahoo.com.br

Submetido em 26/09/2006

Versão final recebida em 08/09/2007 Aceito em 01/11/2007

\begin{abstract}
RESUMO
A marcha é a atividade mais comum que o ser humano realiza, sendo uma habilidade motora fundamental para a locomoção. Contudo, o processo do envelhecimento é caracterizado pela redução gradativa da eficiência do aparelho locomotor, que ocorre pela diminuição da força e da massa muscular, assim como diminuição na flexibilidade. $\mathrm{O}$ estudo objetivou verificar o efeito transiente de uma sessão de exercícios de flexibilidade dos músculos extensores e flexores do quadril sobre a marcha de indivíduos idosos. Cinco idosas (67,0 $\pm 3,8$ anos; $1,59 \pm 0,07 \mathrm{~m} ; 64,3 \pm 15,3 \mathrm{Kg}$ ) voluntariaram participar do estudo. A marcha dos sujeitos foi analisada antes e depois dos exercícios de flexibilidade. O protocolo consistiu em uma sessão de três séries de exercícios de flexibilidade de 30s do método estático, feitos para os músculos flexores e extensores da articulação do quadril. Após a sessão de flexibilidade, a marcha das participantes apresentou menor pico de inclinação anterior da pelve, maior pico de extensão e amplitude total de movimento da articulação do quadril, maior amplitude de movimento do joelho com maior ângulo de flexão durante a fase de balanço médio e maior altura de separação do pé ao solo (aumento esse de 28,6\%). Os resultados mostram que imediatamente após a sessão dos exercícios de flexibilidade as mulheres idosas apresentaram mudanças no padrão da marcha nos quais algumas variáveis sugerem uma redução no risco de quedas. Os efeitos da idade sobre determinadas variáveis foram parcialmente revertidos e as participantes apresentaram um padrão da marcha mais similar aos adultos jovens que antes dos alongamentos.
\end{abstract}

Palavras-chave: terceira idade; alongamento; caminhada e risco de quedas.

\begin{abstract}
Gait is the most common activity performed by humans, being a fundamental motor ability to locomotion. However, ageing is characterized by a gradual reduction of the locomotor apparatus efficiency which occurs by the decrease in strength, muscle mass and flexibility. This study aimed to verify the transient effect of a single session of hip extensor and flexor muscles stretching exercises over gait in elderly subjects. Five elderly ladies (67.0 \pm 3.8 years $1.59 \pm 0.07 \mathrm{~m} ; 64.3 \pm 15.3 \mathrm{Kg}$ ) volunteered to participate in the study. Gait analysis was performed before and after stretching exercises. The stretching protocol was applied in a single session and consisted of three static stretching exercises conducted during 30s for the hip joint flexor and extensor muscles. After the stretching session, participants' gait showed reduced pelvic anterior tilt peak, greater hip range of motion and extension peak, greater knee range of motion, greater knee peak flexion angle during the mid swing and greater toe clearance (28.6\%). Results showed that immediately after a stretching session, the elderly women presented alterations in the gait pattern in which some variables suggest a reduced risk of fall. The effects of ageing over some variables were partially reversed and the participants presented a gait pattern more similar to young adults than before stretching.
\end{abstract}

Keywords: ageing, stretching, gait and risk of falls.

\section{INTRODUÇÃO}

A marcha é a atividade mais comum que o ser humano realiza(1), sendo uma habilidade motora fundamental para a locomoção, automatizada aproximadamente aos sete anos de idade ${ }^{(2)}$ e permanecendo relativamente estável por quase toda a vida(1). Contudo, o processo do envelhecimento é caracterizado pela redução gradativa da eficiência do aparelho locomotor, que ocorre pela diminuição da força e da massa muscular, assim como diminuição na flexibilidade ${ }^{(3)}$. Tais alterações podem modificar os padrões coordenativos de atividades $\operatorname{cotidianas}^{(1,4,5)}$. Alguns estudos têm demonstrado que o padrão da marcha em idosos difere do padrão encontrado em adultos jovens $s^{(2,4,5)}$. Parte dessas diferenças, relacionada à flexibilidade, está ligada à perda progressiva da amplitude das articulações, que ocorre principalmente pela degradação dos tecidos conjuntivos periarticulares ${ }^{(4)}$, considerado com um dos importantes fatores limitantes da flexibilidade músculotendíneo nos idosos ${ }^{(6)}$.

Estudos têm procurado demonstrar que a flexibilidade pode constituir em um fator limitante no desempenho da marcha, especialmente de pessoas idosas. $\mathrm{O}$ encurtamento e a fraqueza dos músculos exten- 
sores do quadril podem limitar sua amplitude de flexão ${ }^{(3,6-9)}$, reduzindo a amplitude articular. Uma redução na flexibilidade da musculatura flexora do quadril compromete o desempenho da mobilização articular - redução da amplitude de hiperextensão articular do quadril e, conseqüente, limitação da amplitude da passada ${ }^{(10-12)}$ - e provoca um aumento na inclinação anterior da pelve durante a marcha, levando a uma instabilidade, devido o deslocamento anterior do centro de gravidade ${ }^{(1,11,12)}$. Essas alterações podem fazer com que a incidência de quedas durante a marcha maior. Berg et al. ${ }^{(13)}$ demonstram que a execução da marcha, independente de velocidade, perfaz um total de $48 \%$ das quedas em idosos. Muito disso é decorrente de tropeços, que ocorrem com mais freqüência em idosos ${ }^{(13)}$, especialmente durante a fase de balanço médio', pois este é o momento onde o pé (na fase aérea) passa mais próximo do chão ${ }^{(14)}$. Estudos confirmam que a maior freqüência e severidade das quedas ocorrem após os 60 anos de idade ${ }^{(15)}$. A queda é a principal causa de mortes acidentais sofrida pelo idoso e estima-se que a cada ano, aproximadamente $20 \%$ da população idosa sofrem de quedas(16).

Desta forma, a manutenção da funcionalidade dos músculos que atuam ao redor da articulação do quadril possui um papel importante sobre o padrão da marcha dos idosos, fazendo-se necessárias estratégias que visem reduzir a perda da flexibilidade ao redor da articulação do quadril. Portanto, este estudo busca verificar o efeito transiente de uma sessão de exercícios de flexibilidade dos músculos extensores e flexores da articulação do quadril sobre a marcha de indivíduos idosos, comparando as alterações de um conjunto de variáveis cinemáticas da marcha em resposta a uma sessão de exercícios de flexibilidade. Os resultados desta investigação podem auxiliar profissionais na área da saúde a melhorar a capacidade funcional e a qualidade de vida na terceira idade.

\section{MÉTODOS}

\section{Sujeitos}

Inicialmente todos os sujeitos foram informados dos procedimentos experimentais e assinaram o termo de consentimento livre e esclarecido, concordando em participar voluntariamente do estudo. O estudo foi aprovado pelo comitê de ética da UFPR. Foram recrutadas cinco mulheres idosas (> 60 anos), aparentemente saudáveis, com média de idade de $67,0(3,8)$ anos, altura de $1,58(0,07)$ metros e peso de $63,7(14,5) \mathrm{kg}$. Nenhum dos sujeitos relatou, por meio de entrevista, problemas conhecidos que pudessem comprometer a marcha (ex.: histórico recente de fraturas, cirurgias articulares, quedas, qualquer tipo de dor articular ou muscular e doença cardiovasculares conhecidas).

\section{Procedimentos Experimentais Gerais}

Os sujeitos compareceram ao laboratório em uma única sessão aonde executaram todo o protocolo experimental. Os sujeitos caminharam no plano da forma mais natural possível por um período de aproximadamente quatro minutos, em velocidade volicional. Após o período de caminhada, os sujeitos foram submetidos a uma série de exercícios de flexibilidade para os músculos extensores e flexores da articulação do quadril. Finalmente, os sujeitos caminharam novamente, durante o mesmo tempo e sob as mesmas condições do pré-teste que precederam à série de exercícios de flexibilidade. Após o segundo procedimento de filmagem, as idosas responderam a uma pergunta fechada sobre sua sensação de bem-estar ou desconforto durante a

\footnotetext{
1 O termo fase de balanço médio corresponde ao termo em inglês "mid-swing", evento da marcha em que o segmento avaliado está na fase aérea com os pés estando lado a lado, aproximadamente a $80 \%$ do ciclo da marcha ${ }^{(1)}$.
}

marcha pós-sessão de exercícios. Havia três opções de resposta: melhor que antes; do mesmo jeito que antes ou pior que antes. O intuito da pergunta era saber, independente dos resultados da análise cinemática, se a caminhada feita logo após os exercícios de flexibilidade foi melhor no conceito das próprias idosas.

\section{Análise cinemática}

Os parâmetros cinemáticos para a análise dos movimentos foram obtidos através de imagens coletadas por uma câmera filmadora digital (JVC GRDVL 9.500 E, New York, USA) em uma freqüência de 50 Hz, posicionada a aproximadamente três metros do hemicorpo direito, no plano sagital focalizado a partir da pelve das participantes. Para que os procedimentos experimentais de filmagem não alterassem o padrão cotidiano da marcha dos idosos, os sujeitos não foram informados sobre o momento de inicio e fim das filmagens. O padrão da marcha antes (PRE) e após (POS) a sessão de flexibilidade foi determinado a partir de uma média agrupada de três ciclos da marcha, obtidos para cada condição experimental. O início e o fim de cada ciclo foram determinados por dois contatos consecutivos do mesmo calcanhar (direito) no solo.

Um conjunto de seis marcadores semi-esféricos, com um diâmetro de $25 \mathrm{~mm}$, foi aderido à pele e roupa por meio de uma fita adesiva dupla-face. Para facilitar a marcação e posterior processamento das marcas, os sujeitos vestiram uma roupa preta justa ao corpo. O conjunto de marcadores foi posicionado sobre os seguintes pontos anatômicos (ver Figura 1): espinha ilíaca ântero-superior (EIAS), trocânter maior (QUADRIL) e epicôndilo lateral do fêmur (JOELHO), maléolo lateral da tíbia (TORNOZELO), aspecto lateral do calcâneo (CALCANHAR) e articulação do quinto metatarso-falangeal (METATARSO). Todos os pontos foram identificados por um único avaliador, com os sujeitos na posição anatômica. A identificação destes pontos anatômicos permitiu definir os seguintes segmentos corporais: borda anterior da pelve (segmento formado pela união da EIAS e o trocânter maior), coxa (segmento formado entre o trocânter maior e epicôndilo lateral do fêmur), perna (segmento formado entre o epicôndilo lateral do fêmur e maléolo lateral da tíbia), pé (segmento formado entre o maléolo lateral da tíbia e o $5^{\circ}$ metatarso-falangeal). A junção dos segmentos descritos fez com que os ângulos das articulações fossem identificados: ângulo do quadril (ângulo formado pela borda anterior da pelve e coxa) e ângulo do joelho (ângulo formado pela junção de coxa e perna).

As variáveis dependentes do estudo utilizadas para a comparação da marcha nas condições experimentais envolveram a análise de um número de parâmetros: pico de inclinação da inclinação da pelve

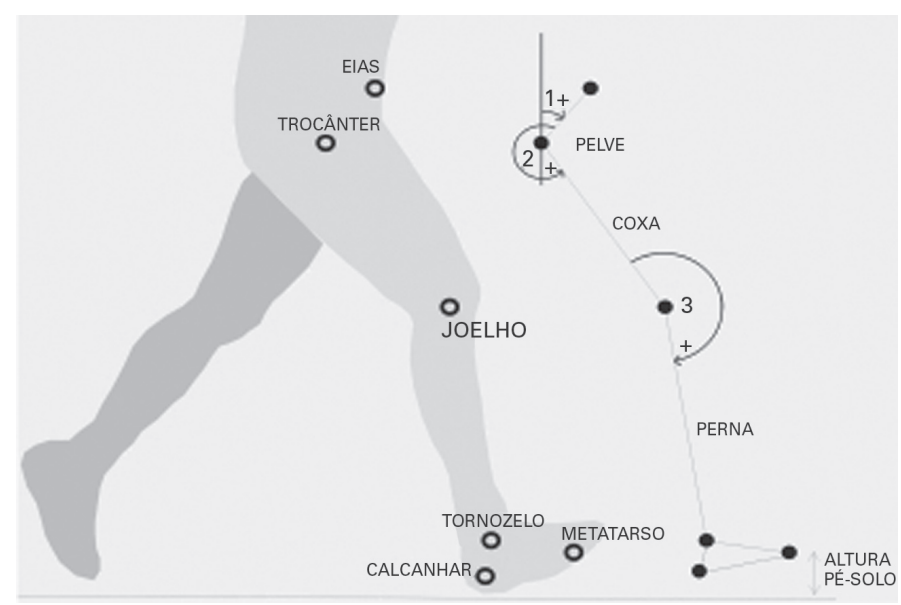

Figura 1. Modelo biomecânico bidimensional

NOTA: 1 - ângulo de inclinação da pelve lateral (relação entre o eixo vertical e o segmento da pelve); 2 - ângulo do quadril; 3 - ângulo do joelho. 
(ponto de máxima inclinação do ângulo da borda anterior da pelve em relação ao eixo vertical); pico de extensão e flexão da articulação do quadril (ponto de máxima extensão e flexão da articulação), amplitude articular do quadril (diferença, em graus, entre o pico de flexão e extensão da articulação), pico de extensão e flexão do segmento da coxa (ponto de máxima extensão e flexão do segmento), amplitude de movimento da coxa (diferença, em graus, entre o pico de flexão e extensão do segmento), pico de flexão do joelho (ponto de máxima flexão da articulação durante a marcha), amplitude articular do joelho (diferença, em graus, entre o pico de flexão e extensão da articulação). Além desses parâmetros da marcha, o comprimento da passada (distância entre dois contatos consecutivos do calcâneo na linha de progressão) e a elevação do pé (altura mínima de separação do metatarso em relação ao solo) foram analisados, mais especificamente na fase do balanço médio(14). Os ângulos da pelve, da coxa e do joelho também foram analisados no momento da fase do balanço médio. Os movimentos de inclinação anterior da pelve e flexão de segmentos e articulações foram considerados como positivos, enquanto que os movimentos de inclinação posterior da pelve e extensão de segmentos e articulações como negativos ${ }^{(1)}$, como mostra a figura 1. A tabela 1 apresenta as variáveis do estudo.

Tabela 1. Variáveis cinemáticas da marcha.

\begin{tabular}{c|l}
\hline Abreviação & \multicolumn{1}{c}{ Variáveis da marcha } \\
\hline PIP & Pico de inclinação da pelve \\
\hline AP $m$ ms & Ângulo da pelve durante o mid-swing \\
\hline PEQ & Pico de extensão da articulação do quadril \\
\hline PFQ & Pico de flexão da articulação do quadril \\
\hline AMQ & Amplitude de movimento do quadril \\
\hline PEC & Pico de extensão do segmento da coxa \\
\hline PFC & Pico de flexão do segmento da coxa \\
\hline AC $m s$ & Ângulo do segmento da coxa durante o mid-swing \\
\hline PFJ & Pico de flexão da articulação do joelho \\
\hline ATMJ & Amplitude de movimento do joelho \\
\hline AJ $m s$ & Ângulo do joelho durante o mid-swing \\
\hline CP & Comprimento da passada \\
\hline AMET & Altura mínima do metatarso em relação ao solo \\
\hline
\end{tabular}

\section{Protocolo do Alongamento}

Após a execução da marcha PRE os sujeitos passaram por um aquecimento padrão (caminhada) de 5 minutos ${ }^{(17)}$, antes da execução do protocolo de flexibilidade. Em seguida, foram submetidos a uma série de exercícios de flexibilidade do tipo estático para os músculos extensores e flexores da articulação do quadril, respectivamente, em ambos os membros inferiores ${ }^{(6)}$. O método de flexibilidade estático foi determinado como padrão, seguindo as recomendações do ACSM ${ }^{(18)}$ que o considerada como método mais adequado para os idosos. Cada exercício teve duração de 30 segundos ${ }^{(8,9,18)}$, sendo repetido três vezes $^{(18)}$, alternando os segmentos (direito e esquerdo), deste modo, o intervalo entre cada série de exercícios foi de 30 segundos.
Para o exercício dos flexores de quadril (Figura 2A) foi utilizado o "teste de Thomas modificado"(19,20). Os sujeitos ficaram em decúbito dorsal sobre uma maca (posição inicial). A coxa do segmento não avaliado foi flexionada a aproximadamente 125 graus, no segmento oposto o avaliador executou uma hiperextensão forçada do quadril e flexão de joelho (posição final). Para o exercício dos extensores de quadril, os sujeitos ficaram em decúbito dorsal sobre uma maca, com ambos os joelhos em extensão (Figura 2B). O avaliador flexionou o quadril do segmento avaliado (com os joelhos em extensão) até ocorrer uma restrição de movimento ou compensação do segmento oposto. Foi aplicada uma força suficiente para promover um movimento lento e gradativo do segmento avaliado(20,21). Depois de terminado o procedimento, os sujeitos executaram a marcha POS.

Ao final da avaliação POS da marcha, foi efetuada uma entrevista direta a respeito da percepção subjetiva de bem-estar após os exercícios de flexibilidade.

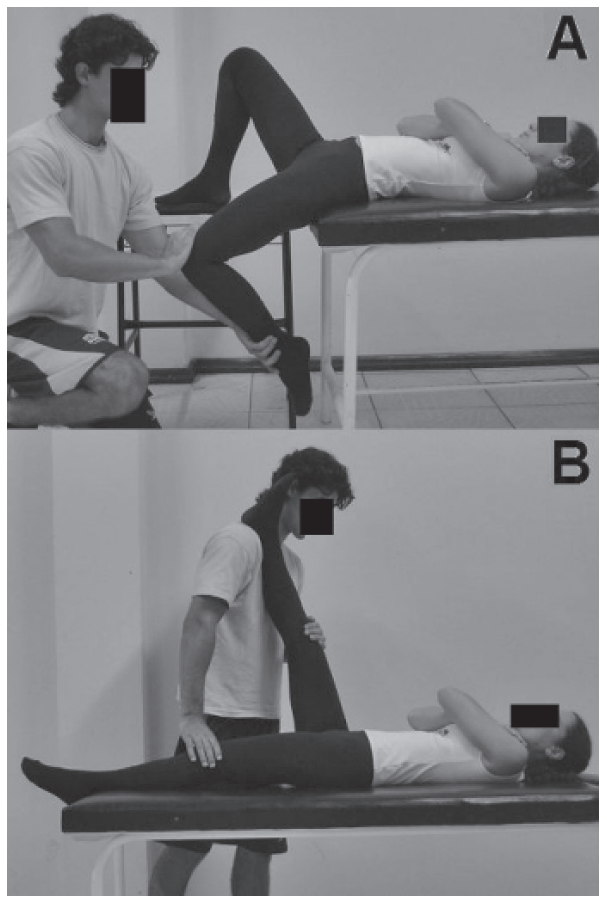

Figura 2. Exercício de flexibilidade para os músculos flexores e extensores do quadril NOTA: Exercício para os músculos flexores do quadril (A) e exercício para os músculos extensores (B).

\section{Análise dos Dados e Tratamento Estatístico}

O software SIMI Motion (versão 6.1, Unterschleissheim, Germany), através de coordenadas cartesianas do conjunto de pontos anatômicos expostos fez a reconstrução do movimento para a obtenção dos dados. Esses dados foram digitalizados e filtrados pelo mesmo software. A análise dos dados foi realizada empregando uma estratégia semelhante à de Mills et al. ${ }^{(14)}$, utilizando um filtro recursivo de segunda ordem do tipo Butterworth, com uma freqüência de $6 \mathrm{~Hz}$.

Os dados obtidos foram submetidos a uma análise descritiva padrão (média e desvio padrão). O teste de Shapiro-Wilks foi aplicado para analisar a normalidade dos dados, além de uma análise inferencial não-paramétrica de Wilcoxon para amostras pareadas. As análises foram realizadas através do software STATISTICA (Statistica, versão 5.5, USA). 0 nível de significância de cada variável foi de $p<0,05$.

\section{RESULTADOS}

Os resultados das variáveis cinemáticas (média \pm desvio padrão) da marcha dos idosos nas condições de pré e pós-exercícios de flexibilidade encontram-se representados na tabela 2 . 
Tabela 2. Valores das variáveis cinemáticas (média \pm desvio padrão) nas condições PRE e POS. * $p<0,05$.

\begin{tabular}{|c|c|c|c|}
\hline Variáveis & Pré & Pós & $P$ \\
\hline PIP (graus) & $6,5 \pm 5,5$ & $1,7 \pm 3,8$ & $0,04^{*}$ \\
\hline PEQ (graus) & $-10,3 \pm 2,8$ & $-14,2 \pm 3,7$ & $0,04^{*}$ \\
\hline PFQ (graus) & $20,8 \pm 5,3$ & $16,1 \pm 2,9$ & $0,04^{*}$ \\
\hline PEC (graus) & $-13,4 \pm 3,6$ & $-14,3 \pm 1,9$ & 0,22 \\
\hline PFC (graus) & $20,5 \pm 2,3$ & $21,4 \pm 1,7$ & 0,22 \\
\hline PFJ (graus) & $55,2 \pm 3,2$ & $56,5 \pm 3,0$ & 0,14 \\
\hline $\mathrm{CP}(\mathrm{m})$ & $0,93 \pm 0,12$ & $0,98 \pm 0,12$ & 0,08 \\
\hline $\operatorname{AMET}(\mathrm{cm})$ & $2,1 \pm 0,01$ & $2,7 \pm 0,03$ & $0,04^{*}$ \\
\hline AMQ (graus) & $31,0 \pm 5,3$ & $29,7 \pm 5,4$ & 0,50 \\
\hline AMC (graus) & $33,9 \pm 1,6$ & $35,7 \pm 1,9$ & $0,04^{*}$ \\
\hline AMJ (graus) & $54,8 \pm 2,3$ & $57,0 \pm 3,2$ & $0,04^{*}$ \\
\hline
\end{tabular}

${ }^{*} p<0,05$

Os valores dos ângulos da pelve e das articulações durante o balanço médio se encontram na tabela 3.

Tabela 3. Valores (média \pm desvio padrão) dos ângulos da pelve, coxa, joelho e tornozelo durante o mid-swing.

\begin{tabular}{c|c|c|c}
\hline Variáveis & Pré & Pós & P \\
\hline$A P_{\text {ms }}$ (graus) & $-2,9 \pm 4,0$ & $-8,8 \pm 1,6$ & $0,04^{*}$ \\
\hline$A C_{m s}$ (graus) & $18,4 \pm 1,8$ & $18,9 \pm 1,2$ & 0,69 \\
\hline$A J_{m s}$ (graus) & $43,3 \pm 7,1$ & $45,3 \pm 6,4$ & $0,04^{*}$ \\
\hline$A T_{m s}$ (graus) & $1,9 \pm 3,6$ & $3,0 \pm 4,8$ & 0,35 \\
\hline
\end{tabular}

NOTA: Valores negativos da $\mathrm{AP}_{\mathrm{ms}}$ representam inclinação posterior da pelve. ${ }^{*} p<0,05$.

Após os exercícios de flexibilidade, os sujeitos apresentaram uma diminuição do PIP de aproximadamente $5^{\circ}(p<0,05)$, como mostra a figura 3. O PEQ apresentou aumentos de 3,9 $(p<0,05)$ e PFQ uma redução de $4,7^{\circ}$, após a execução dos exercícios de flexibilidade (Figura 4). A AMC (Figura 5) mostrou um aumento de 1,80 após a série de exercícios $(p<0,05)$.

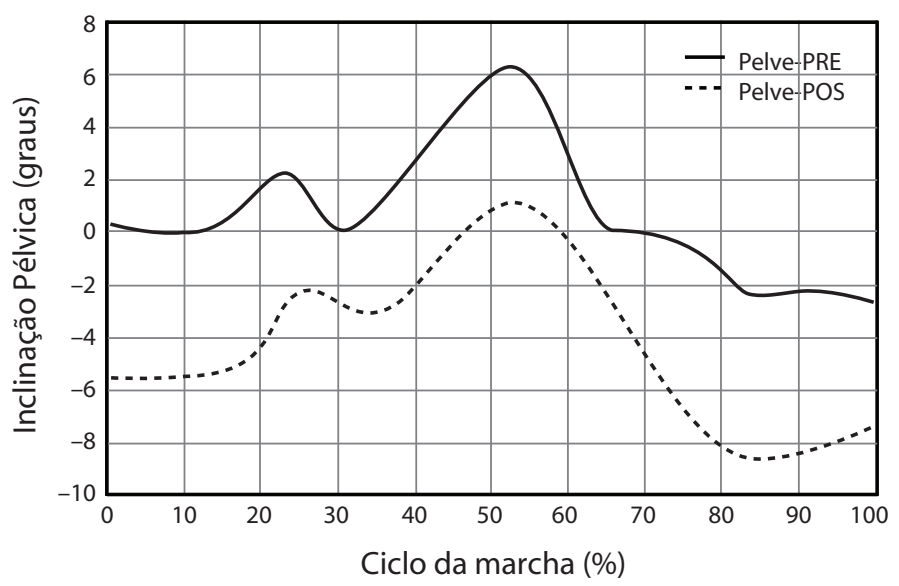

Figura 3. Variação angular da pelve (PIP) antes (PRE) e após (POS) os exercícios de flexibilidade.

NOTA: Os valores positivos indicam maior inclinação anterior da pelve

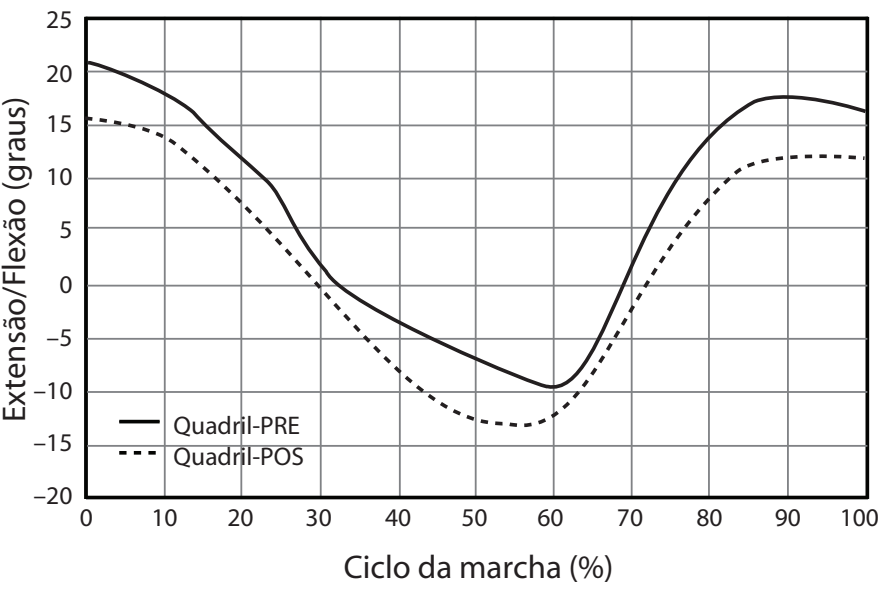

Figura 4. Variação angular do quadril (PEQ e PFQ) antes (PRE) e após (POS) Os exercícios de flexibilidade.

NOTA: Os valores de extensão são considerados como negativos e os de flexão positivos.

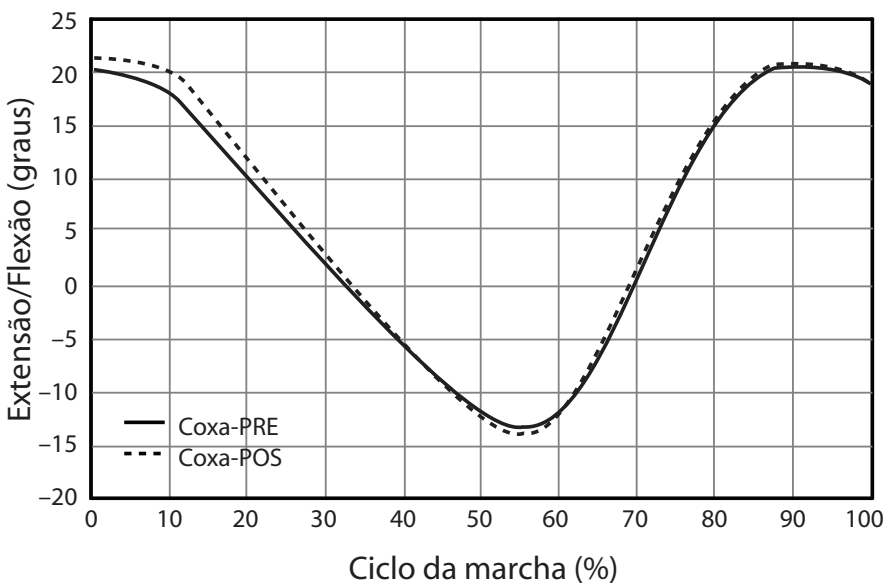

Figura 5. Variação angular da coxa (PEC e PFC) antes (PRE) e após (POS) os exercícios de flexibilidade.

NOTA: Os valores de extensão são considerados como negativos e os de flexão positivos.

A PFJ também não mostrou ser sensível aos exercícios de flexibilidade $(p>0,05)$. A amplitude de movimento na articulação do joelho aumentou $2.2^{\circ}(p<0,05)$.

O comprimento da passada (CP) não aumentou em função dos exercícios de flexibilidade $(p=0,08)$. A altura mínima de separação do pé ao solo (AMET), PRE e POS os exercícios de flexibilidade, demonstrou diferenças significativas $(0,021 \pm 0,001 \mathrm{~m}$ para 0,027 $\mathrm{m} \pm 0,003 \mathrm{~m}$, respectivamente, $p<0,05)$.

No momento que a AMET foi calculada, durante a fase de balanço médio, o $\mathrm{AP}_{\mathrm{ms}}$ obteve uma maior diminuição de sua inclinação anterior $\left(5,9^{\circ}\right)$ e $\circ \mathrm{AJ}$ ms um aumento na flexão $\left(2,0^{\circ}\right)$ de maneira significativa $(p>0,05)$ após a execução dos exercícios de flexibilidade (Tabela 3$)$.

Quanto à pergunta feita após a avaliação POS da marcha, 100\% das idosas reportaram uma sensação de bem-estar ao caminhar após os exercícios de flexibilidade.

\section{DISCUSSÃO}

A manutenção da habilidade de caminhar é importante para sujeitos idosos, pois constitui um elemento fundamental em todas as suas atividades diárias independentes ${ }^{(2)}$. A manutenção das funções 
normais da marcha é um fator determinante para a redução do risco de quedas entre idosos ${ }^{(22,23)}$. O presente estudo objetivou verificar a influência do efeito transiente de exercícios de flexibilidade sobre um conjunto de variáveis cinemáticas que descrevem a marcha. Os efeitos dos exercícios de flexibilidade sobre os grupos musculares não foram quantificados, porém alguns estudos ${ }^{(7-9)}$ que utilizaram rotinas similares àquela aplicada no presente estudo, demonstraram que tais atividades são efetivas e causam alterações sobre a amplitude dos músculos que cruzam as articulações do quadril e joelho. Em indivíduos idosos, espera-se que tais rotinas de flexibilidade tenham efeitos ainda mais pronunciados, visto que as reduções nas amplitudes articulares decorrentes de encurtamentos músculo-tendíneos em idosos são mais acentuadas que em jovens $s^{(3,4,6)}$.

Independente do tipo, ou mesmo das características dos exercícios empregados na sessão de flexibilidade, tais exercícios foram satisfatórios para provocar alterações em um número de variáveis dinâmicas da marcha. Dentre as alterações induzidas pelos exercícios de flexibilidade, o aumento da altura do metatarso em relação ao solo foi a mais importante. Aumentos na altura mínima de elevação do metatarso em relação ao solo são determinantes para a redução do risco de tropeços e quedas em idosos ${ }^{(1,23,24)}$. Os valores da altura mínima de elevação do metatarso registrados antes dos exercícios de flexibilidade são compatíveis os dados populacionais reportados por Mills et al. ${ }^{(14)}$ que descreveram alturas de aproximadamente 0,02 $\mathrm{m}$ em uma população com faixa etária similar (68,9 anos) àquela empregada no presente estudo. Os ganhos na altura de elevação do metatarso em relação ao solo representaram um aumento de $28,57 \%(2,1 \mathrm{~cm}$ $-2,7 \mathrm{~cm}$ ) e podem constituir um importante fator na redução no risco de quedas em populações de idosos. Estudos que tenham comparado esta variável em sujeitos idosos após exercícios de flexibilidade não são conhecidos, portanto, não foi possível comparar os achados deste estudo com outros. O aumento da altura mínima do metatarso não pode ser explicado por diferenças nos movimentos da articulação do quadril, que permaneceu inalterada entre as condições experimentais, no instante em que a mínima elevação do metatarso foi quantificada. Portanto, entende-se que após os exercícios de flexibilidade, a menor inclinação anterior da pelve e maior flexão da articulação do joelho permitiram uma maior altura do pé em relação ao solo. No presente estudo, o ângulo de flexão do joelho no instante do balanço médio foi similar aos valores reportados em estudos com idosos ${ }^{(14)}$, todavia a comparação do ângulo de inclinação anterior da pelve não foi possível pela falta de estudos que reportem esta variável no instante do balanço médio. Desta forma, a redução na limitação da inclinação posterior da pelve dos sujeitos idosos (causada pelo encurtamento dos músculos flexores do quadril) também pode explicar a facilitação na execução do movimento, permitindo que as idosas elevem a altura de todo o membro inferior, produzindo um aumento na altura mínima do metatarso em relação ao solo.

Os aumentos no pico de flexão e na amplitude de movimento do joelho após os exercícios de flexibilidade foram evidenciados Tanto a maior flexão durante a mínima altura do metatarso em relação ao solo, como a alteração na amplitude da articulação do joelho pode estar associada a uma menor resistência que os músculos flexores do joelho (ísquio-tibiais) possam ter encontrado na fase de balanço da marcha. Aumentos no comprimento na unidade músculo-tendínea do músculo reto femoral decorrente dos exercícios de flexibilidade podem explicar tais aumentos, uma vez que melhorias na capacidade dos músculos flexores do joelho em mover esta articulação não são esperadas. Outros estudos são necessários para testar esta possibilidade. Tais alterações mostram que após os exercícios de flexibilidade, a menor amplitude dinâmica de flexão do joelho na fase aérea, fosse aumentada. Murray et al..$^{(5)}$ apontam que a flexão do joelho é uma das limitações funcionais mais evidenciadas na marcha de idosos. Essa alteração fez com que a altura mínima do metatarso em relação ao solo durante a fase de balanço médio também fosse influenciada.

As alterações ocorridas na pelve (pico de inclinação anterior) e na altura mínima do metatarso em relação ao solo sugerem que os músculos flexores da articulação do quadril sofreram alterações funcionais após os exercícios de flexibilidade. Ao analisar o ângulo da coxa, que não sofreu alterações significativas (em torno de $1^{\circ}$ para o pico de flexão e extensão), conclui-se que os exercícios de flexibilidade afetaram mais pronunciadamente os músculos que controlam os movimentos de inclinação da pelve (ex:: músculo ílio-psoas).

Acredita-se que o aumento no comprimento músculo-tendíneo do reto femoral e do ílio-psoas durante o mid-swing influenciou somente a articulação do joelho e o segmento da pelve, respectivamente, devido à necessidade da articulação do quadril trabalhar como um ponto de equilíbrio durante a execução da marcha. Sem isso, o movimento poderia acabar sendo executado com menos controle e maior instabilidade. Essa função do quadril atuando como um eixo se explica na hipótese onde uma das inserções de ambos os músculos (as que influenciariam especificamente o quadril) atuasse como ponto mais fixo. A modificação encontrada no reto femoral após os exercícios de flexibilidade teve efeito somente no joelho, além da relação do quadril posta acima, pois esse músculo exerce menos influencia sobre a flexão do quadril do que o músculo ílio-psoas, atuando mais vigorosamente sobre a extensão do joelho.

Em geral, os reduzidos valores encontrados no pico de extensão do quadril em idosos em comparação a sujeitos jovens, têm sido associados a um aumento na inclinação anterior da pelve ${ }^{(1,25)}$. Aumentos no pico de extensão do quadril e na inclinação da pelve indicam uma redução no comprimento funcional da unidade músculo-tendínea ("contratura") dos músculos flexores que atuam ao redor do quadril e têm sido descritas como decorrentes do processo de envelhecimento ${ }^{(10)}$. O presente estudo demonstrou que rotinas de flexibilidade podem modificar de maneira transiente o comprimento da unidade músculo-tendínea dos flexores do quadril e produzir um maior ângulo de extensão da articulação. Comparando as figuras 3 e 5, vê-se que as alterações ocorridas na articulação do quadril (Figura 4) estão, em grande parte, associadas a menor inclinação anterior da pelve, que rodou em sentido anti-horário diminuindo o ângulo de flexão e extensão desta articulação. Esse fato pode ser explicado, pois outros estudos mostram que, mesmo de maneira não significativa, os adultos jovens apresentam uma maior extensão e menor flexão do quadri|(10,12). Kerrigan et al.(10) têm reportado aumentos de $5^{\circ}$ no ângulo de extensão do quadril em idosos saudáveis quando comparados a sujeitos jovens. Este ângulo é ainda mais acentuado (90) em idosos com histórico de queda ${ }^{(10)}$. Portanto, é possível afirmar que rotinas de flexibilidade podem causar alterações no comprimento músculo-tendíneo fazendo com que os idosos apresentem padrões de marcha mais similares aos adultos jovens, reduzindo o risco de quedas associado à terceira idade.

Os resultados do presente estudo não confirmam os achados de Kerrigan et al. ${ }^{(11)}$, que realizaram uma rotina de exercícios de flexibilidade para os flexores do quadril durante 10 semanas em sujeitos idosos. Os resultados de Kerrigan et al. ${ }^{(11)}$ demonstraram uma tendência (não 
significativa) de redução da inclinação anterior da pelve, que resultaram em uma modesta melhora na extensão do quadril. A falta de controle sobre o protocolo de flexibilidade pode ter comprometido a efetividade do programa. Além disso, os exercícios de flexibilidade utilizados no presente estudo não incluíram apenas os grupos musculares flexores, mas também os músculos extensores da articulação do quadril. Um maior comprimento músculo-tendíneo dos extensores do quadril pode facilitar o alcance do membro contra-lateral durante a fase de balanço e melhorar os aspectos dinâmicos da marcha, como a AMC e o PEQ. Isso fica claro quando as pesquisas relatam que a redução na extensão do quadril, em parte pela inclinação anterior da pelve, especialmente no plano sagital(26), pode ser o mecanismo fundamental de diminuição do comprimento da passada da marcha em idosos ${ }^{(10,11,25)}$. De fato, foi observada uma tendência dos sujeitos em demonstrarem uma amplitude do ciclo da marcha mais acentuada. Independente de o resultado ser quase significativo.

De fato que os exercícios de flexibilidade do método estático, com 30 segundos de duração, feitos de maneira supervisionada e com uma avaliação posterior realizada dentro do limite de recuperação músculo-tendínea ${ }^{(27)}$ têm efeitos positivos, não só sobre a amplitude de movimento passiva das articulações ${ }^{(8,9,27)}$, mas também sobre a marcha. Então, deve-se entender a importância da supervisão sobre a execução dos exercícios, pois como King et al. ${ }^{(28)}$ relatam que a falta do mesmo causa a diminuição da adesão à atividade física e dos resultados conquistados. Além disso, não foi observado no estudo qualquer efeito negativo dos exercícios de flexibilidades sobre os sujeitos, pelo contrário, todas reportaram uma maior sensação de bem-estar durante a caminhada após a sessão de flexibilidade. Desta forma, uma sessão de exercícios de flexibilidade feitos de maneira adequada em sujeitos idosos promoveu alterações significativas no comportamento da pelve, quadril, joelho e altura de separação do pé do solo, fazendo com que a marcha desta faixa etária seja executada de forma mais segura.

Como se trata de um estudo bidimensional, certas variáveis cinemáticas não podem ser calculadas, como a inclinação lateral e rotação da pelve. Futuros estudos necessitam avaliar a relação entre marcha e exercícios de flexibilidade em um plano tridimensional.

Concluiu-se que os exercícios de flexibilidade ocasionaram importantes modificações transientes (agudas) em diversas variáveis cinemáticas na marcha das idosas, das quais se pode ressaltar uma maior elevação do metatarso em relação ao solo. Portanto, os resultados sugerem que logo após uma sessão de exercícios de flexibilidade, os sujeitos idosos executaram a marcha de maneira mais segura fazendo com que quedas, proveniente principalmente de tropeços, possam ser reduzidas. Para que essas alterações provem ser permanentes, esperase que outros estudos sejam feitos com um grupo maior de sujeitos analisando tais efeitos dos exercícios de flexibilidade separadamente e/ou em longo prazo (crônico).

Todos os autores declararam não haver qualquer potencial conflito de interesses referente a este artigo.

\section{REFERÊNCIAS BIBLIOGRÁFICAS}

1. Winter DA. The biomechanical and motor control of human gait: normal, elderly and pathological. 2nd. Waterloo: University of Waterloo Press, 1991.

2. Viel E. A marcha humana, a corrida e o salto: biomecânica, investigações, normas e disfunções. Paris: Masson, 2000. Trad. Maria Alice Farah Calil Antonio. Barueri: Manole, 2001.

3. Shagold M, Mirkin G. Physiology and sports medicine. 2 nd. Philadelphia: F. A. Davis Company, 1994.

4. Prince F, Corriveau H, Hébert R, Winter DA. Gait in the elderly. Gait and Posture 1997; 5: 128-35.

5. Murray MP, Kory RC, Clarkson BH. Walking patterns in healthy old men. J of Gerontol 1969; 24: $169-78$.

6. Feland JB, Myrer JW, Schulthies SS, Fellingham GW, Measom GW. The effect of duration of stretching of the hamstring muscle group for increasing range of motion in people aged 65 years or older. Phys Ther 2001; 81: 1110-7.

7. Feland JB, Myrer JW, Merrill RM. Acute changes in hamstring flexibility: PNF versus static stretch in senior athletes. Phy Ther Sport 2001; 2: 186-93.

8. Bandy WD, Irion JM. The effect of time on static stretch on the flexibility of the hamstrings muscles. Phys Ther 1994; 74: 845-52.

9. Bandy WD, Irion JM, Briggler M. The effect of time and frequency of static stretching on flexibility of the hamstrings muscles. Phys Ther 1997; 77: 1090-6.

10. Kerrigan DC, Lee LW, Collins JJ, Riley PO, Lipsitz LA. Reduced hip extension during walking: health elderly and fallers versus young adults. Arch Phys Med Rehab 2001; 82: 26-30.

11. Kerrigan DC, Xenopoulos-Oddsson A, Sullivan MJ, Lelas JJ, Riley PO. Effect of a hip flexor-stretching program on gait in elderly. Arch Phys Med Rehab 2003; 84: 1-6.

12. Kerrigan DC, Todd MA, Croce UD, Lipsitz LA, Collins JJ. Biomechanical gait alterations independent of speed in the healthy elderly: evidence for specific limiting impairments. Arch Phys Med Rehab 1998; 79: $317-22$.

13. Berg WP, Alessio HM, Mills EM. Circumstances and consequences of falls independent communitydwelling older adults. Age and Aging 1997; 26: 261-8.

14. Mills PM, Barrett RS. Swing phase mechanics of healthy young and elderly men. Hum Mov Sci 2001; 20: $427-46$.
15. American Geriatrics Society. Guideline for the prevention of falls in older person. J Am Geront Soc 2001; 49: 664-72.

16. Gehlsen GM, Whaley MH. Falls in the elderly: part I, gait. Arch Phys Med Rehab 1990; 71: 735-8.

17. Allerheiligen WB. Stretching and Warm-up. In: Baechle TR. Essentials of strength training and conditioning. Illinois: Human Kinetics. 1994; 289-313.

18. ACSM. ACSM's guidelines for exercise testing and prescription. 5 th ed. USA: Williams \& Wilkins, 1995.

19. Sarraf TA, Dezan VH, Rodacki ALF. Diferenças entre medidas quali e quantitativas durante testes de comprimento músculo-tendíneos dos flexores do quadril uni e biarticulares. Revista Brasileira de Fisioterapia 2005; 9: 195-202.

20. Kendall FP, Mccreary EK, Provance PG. Músculos provas e funções. São Paulo: Manole, 1995.

21. Dezan VH, Sarraf TA, Rodacki ALF. Alterações posturais, desequilíbrios musculares e lombalgias em atletas de luta olímpica. Revista Brasileira de Ciência e Movimento 2004; 12: 35-8.

22. Honeycutt PH, Ramsey P. Factors contributing to falls in elderly men living in the community. Geriatric Nurs 2002; 23: 250-7.

23. Woollacott M, Shumway-cook A. Attention and control of posture and gait: a review of an emerging area of research. Gait and Posture 2002; 16: 1-14.

24. Buzzi UH, Stergiou N, Kurz MJ, Hageman PA, Heidel J. Nonlinear dynamics indicates aging affects variability during gait. Clin Biom 2003; 18: 435-43.

25. Riley PO, Dellacroce U, Kerrigan DC. Effect of age on lower extremity joint moment contributions to gait speed. Gait and Posture 2001; 14: 264-70.

26. Hirose $D$, Ishida K, Nagano $Y$, Takahashi T, Yamamoto $H$. Posture of trunk in the sagittal plane is associated with gait in community-dwelling elderly population. Clin Biom 2004; 19: 57-63.

27. Spernoga SG, Uhl TL, Arnold BL, Gansneder BM. Duration of maintained hamstring flexibility after a one-time, modified hold-relax stretching protocol. J Athl Train 2001; 36: 44-8.

28. King MB, Whipple RH, Gruman CA, Judge JO, Schmidt JA, Wolfson LI. The performance enhancement project: improving physical performance in older persons. Arch Phys Med Rehab 2002; 83: 1060-9. 